\title{
Accurate Determination of Grain Boundary Coverages of Segregating Elements by STEM X-ray Mapping Combined with the $\zeta$-Factor Method
}

\author{
M. Watanabe and D.B. Williams \\ Dept. of Materials Science and Engineering, Lehigh University, Bethlehem, PA18015, USA
}

Grain boundary segregation is primarily characterized by the measuring the composition of the segregating element. By using a scanning transmission electron microscope combined with an Xray energy dispersive spectrometer (STEM-XEDS), the compositions of segregants around interfaces can be routinely measured by point analysis or line profiling. One of the best approaches to measure local composition changes around boundaries is X-ray mapping since segregant distributions not only in the vicinity of the boundaries but also along the boundaries can be obtained. Currently, high spatial-resolution X-ray mapping in STEM has been performed for studies of grain-boundary segregation in $\mathrm{Cu}-\mathrm{Bi}$ [1], $\mathrm{Cu}-\mathrm{Sb}$ [2] and $\mathrm{Al}-\mathrm{Cu}$ [3] systems at Lehigh. Unfortunately, the practically measured compositions of boundary segregants cannot always be a reliable quantitative measure of boundary segregation [4]. This is because the measured compositions of segregants are influenced by the specimen thickness, the boundary inclination, the incident beam sizes for analysis and the other experimental conditions. The boundary coverage $\Gamma^{\mathrm{ex}}$, which is the number of excess atoms per unit area in the boundary plane, is a better measure to characterize the boundary segregation because the boundary coverage is less sensitive to the specimen and experimental conditions. Therefore, $\Gamma^{\mathrm{ex}}$ is more reliable especially in comparison with other experimental approaches. This paper presents an approach to determine the boundary coverage in STEM-XEDS more accurately.

From the compositions of the solute element $\mathrm{A}$ and the major element $\mathrm{B}, \mathrm{C}_{\mathrm{A}}$ and $\mathrm{C}_{\mathrm{B}}$, which are the primary results obtained by STEM-XEDS, the boundary coverage for A can be determined using the following equation:

$$
\Gamma_{\mathrm{A}}^{\mathrm{ex}}+\Gamma_{\mathrm{A}}^{\mathrm{bk}}=\mathrm{N}_{\mathrm{B}} \frac{\mathrm{C}_{\mathrm{A}}}{\mathrm{C}_{\mathrm{B}}} \frac{\mathrm{A}_{\mathrm{B}}}{\mathrm{A}_{\mathrm{A}}} \frac{\mathrm{V}}{\mathrm{A}}
$$

where $\Gamma^{\mathrm{bk}}$ is the number of solute atoms per unit area in the bulk, $\mathrm{N}_{B}$ is the number of $B$ atoms per unit volume in the surrounding bulk region, $A_{A}$ and $A_{B}$ are the atomic weights for $A$ and $B$, and $\mathrm{V}$ and $\mathrm{A}$ are the interaction volume and the area of the boundary inside the interaction volume, respectively. Because knowledge of $\mathrm{V}$ and $\mathrm{A}$ (which are dependent on the specimen thickness) is required, it is essential to obtain the specimen thickness for accurate determination of $\Gamma^{\mathrm{ex}}$. Recently, a new quantification technique for thin specimens, called the $\zeta$-factor method, has been developed $[5,6]$. By the $\zeta$-factor method, specimen composition and thickness at the measured point can be determined simultaneously only from XEDS data. Therefore, by X-ray mapping in combination with the $\zeta$-factor method, it is feasible to determine the V/A term in the above equation, and hence to extract the requisite information on the boundary coverage.

An annular dark-field (ADF) image and corresponding thickness map and compositional maps of solute elements (Cr, Ni and Mo) around a prior austenite grain-boundary in a low-alloy steel, taken by a VG HB 603 STEM at $300 \mathrm{keV}$, are shown in Figs. 1(a), (b) and (d)-(f) respectively. The compositional maps show that $\mathrm{Cr}$, $\mathrm{Ni}$ and Mo solute elements are co-segregated on the boundary. It can also be seen that compositions of these elements seem to fluctuate, even on the same boundary. Since measured composition can be influenced by both the specimen and experimental conditions as mentioned above, the compositional fluctuations of the segregants on the boundary may not be truly present. Fig. 1 (c) shows the V/A map calculated from the measured composition and thickness. The V/A value changes locally around the boundary. From 
the local V/A value, the boundary coverages of $\mathrm{Cr}$, Ni and Mo were determined at each pixel, as shown in Fig. (g)-(i), respectively. The coverage maps are more reliable indicators of segregation behavior. The boundary coverage of $\mathrm{Ni}$ is rather homogeneous, but the coverage of $\mathrm{Cr}$ and Mo appear different. The difference in the boundary coverages of $\mathrm{Cr}$ and Mo can be related to discontinuous carbide formation on the boundary. Thus, X-ray mapping combined with the $\zeta$ factor method provides hitherto unobtainable, spatially resolved data about segregation behavior.

\section{References}

[1] V.J. Keast and D.B. Williams, Acta Mater., 47 (1999), 3999.

[2] C. Li et al., Mater. Leters., 57 (2003), 1345.

[3] D.T. Carpenter et al., Microscopy \& Microanalysis, 5 (1999), 254.

[4] V.J. Keast and D.B. Williams, J. Microscopy, 199 (2000), 45.

[5] M. Watanabe et al., Ultramicroscopy 65 (1996), 187.

[6] M. Watanabe and D.B. Williams, Microscopy \& Microanalysis 5 (1999) Suppl. 2, 88.

[7] This research was supported by Bechtel Bettis Laboratories and NSF DMR 99-72670.

(a) STEM-ADF

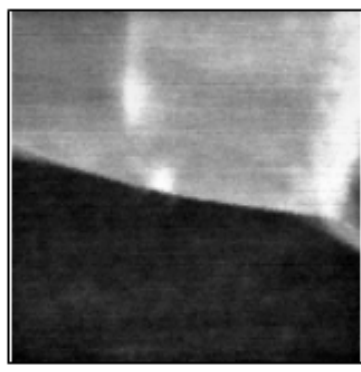

(d) $\mathrm{Cr}(\mathbf{w t} \%)$

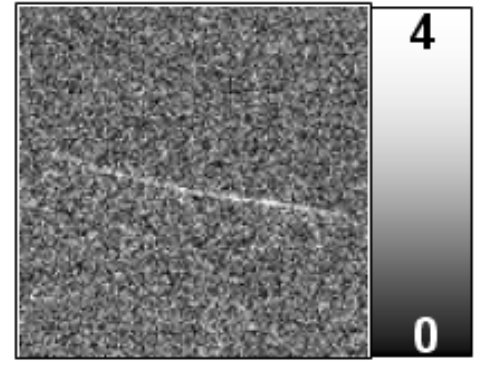

(g) $\Gamma_{\mathrm{Cr}}^{\mathrm{ex}}\left(\right.$ atoms $\left./ \mathrm{nm}^{2}\right)$

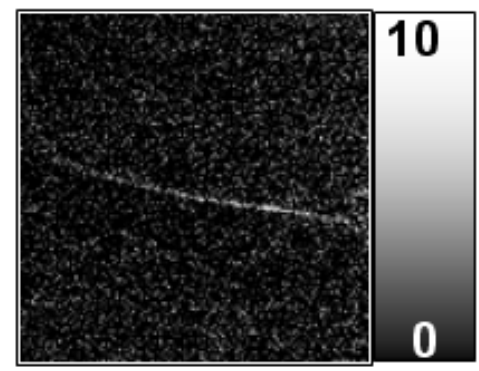

(b) thickness (nm)

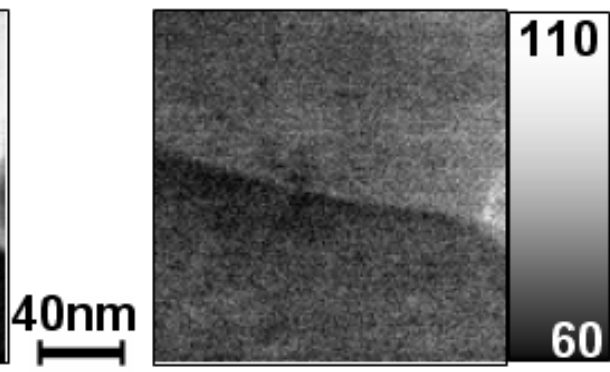

(e) $\mathrm{Ni}(\mathrm{wt} \%)$

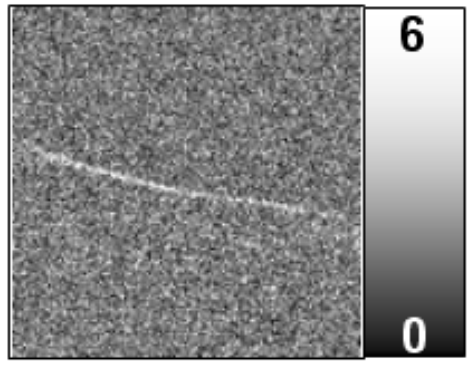

(h) $\Gamma_{\mathrm{Ni}}^{\mathrm{ex}}\left(\right.$ atoms $\left./ \mathrm{nm}^{2}\right)$

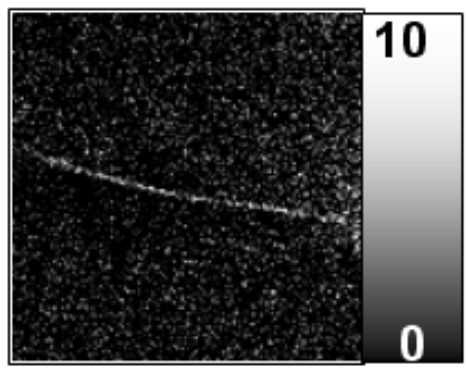

(c) $V I A(\mathrm{~nm})$

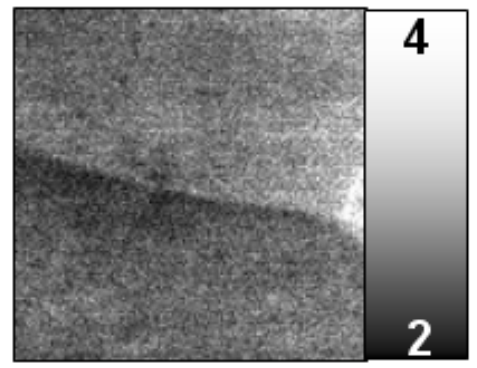

(f) Mo (wt\%)

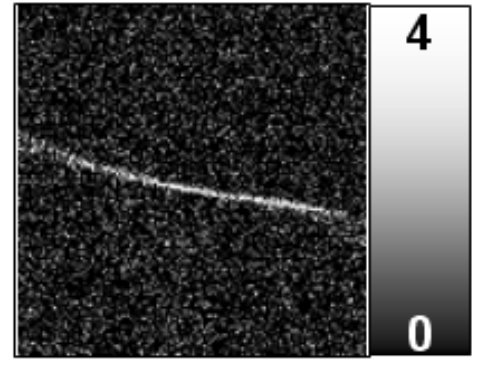

(i) $\Gamma_{\mathrm{Mo}}^{\mathrm{ex}}\left(\right.$ atoms $\left./ \mathrm{nm}^{2}\right)$

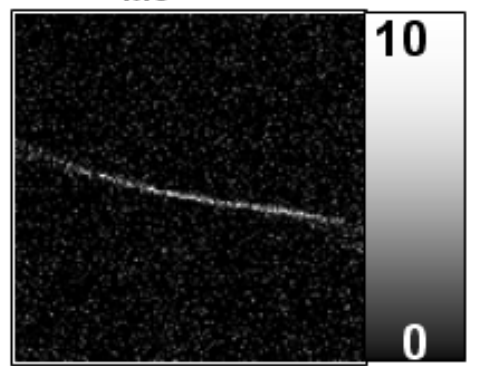

Fig. 1. The ADF-STEM image (a), thickness map (b) and compositional maps of Cr (d), Ni (e) and Mo (f), obtained around a prior austenite grain-boundary in a low-alloy steel. The corresponding V/A (c) and boundary coverage maps of $\mathrm{Cr}(\mathrm{g}), \mathrm{Ni}(\mathrm{h})$ and $\mathrm{Mo}(\mathrm{i})$ were determined by the $\zeta$-factor method. 